\title{
Purity distribution for bipartite random pure states
}

\author{
Olivier Giraud \\ Laboratoire de Physique Théorique, Université Toulouse III, CNRS, 31062 \\ Toulouse, France \\ E-mail: giraud@irsamc.ups-tlse.fr
}

\begin{abstract}
Analytic expressions for the probability density distribution of the linear entropy and the purity are derived for bipartite pure random quantum states. The explicit distributions for a state belonging to a product of Hilbert spaces of dimensions $p$ and $q$ are given for $p=3$ and any $q \geq 3$, as well as for $p=q=4$.
\end{abstract}

PACS numbers: 03.67.Mn, 03.67.-a 


\section{Introduction}

Characterizing entanglement is one of the challenging issues that has been fostered by the development of quantum computation in the past few years. Beyond the obvious interest for the foundations of quantum mechanics, the motivations increased as the role played by entanglement in the power of quantum algorithms was made clearer.

As random states are entangled with high probability, they play an important role in the field of quantum communication, and appear in many algorithms, such as quantum data hiding protocols [1] or superdense coding [2]. Various quantum algorithms were proposed to generate random states: algorithms based on the entangling power of quantum maps have been proposed in [3] chaotic maps were considered for instance in 4, 5, pseudo-integrable maps in 6]. It was also proposed to generate random states by construction of pseudo-random operators [7, or by sequences of two-qubit gates [8]. The efficiency of all these algorithms is measured by their ability to reproduce the entangling properties of random states. Thus, it is crucial to have some measure of entanglement.

The problem of quantifying entanglement is a difficult issue, and a number of entanglement measures have been proposed (see e.g. the review [9]). However for pure quantum states, bipartite entanglement, that is the entanglement of a subset of the qubits with the complementary subset, is essentially measured by the von Neumann entropy of the reduced density matrix [10]. More convenient to use are the linear entropy or the purity, which are linearized versions of the von Neumann entropy. Purity or linear entropy were used to estimate the entangling properties of chaotic quantum maps (for instance in the Baker's map [5] or in kicked tops [11]), or entanglement growth under random unitary evolution [12]. It has been used as a reference to compare the accuracy of various entanglement measures [13, or to measure dynamical generation of entanglement in coupled bipartite systems [14. Recently, it was shown that purity for a pure quantum state could be expressed as a function of the inverse participation ratio, thus enabling to connect entanglement (as measured by the purity) to localization properties of quantum states [15, 16].

In most of these works, the entanglement properties of the systems that are studied are compared to those of random pure states. This is usually done by resorting to numerical computations, based e.g. on Hurwitz parametrization 17. of random states. Of course, a comparison directly based not on numerics but on analytical formulae describing the entanglement properties of random pure states would be most desirable. In this paper we derive such formulae for the purity and the linear entropy.

Random pure states can be realized as column vectors of random unitary matrices drawn from an ensemble with unitarily invariant Haar measure [18. Equivalently they can be realized as vectors with coefficients given by independent random complex Gaussian variables, rescaled to have a norm equal to 1 (see e.g. 19]). Various aspects of the entanglement properties of random pure states have been studied in previous works: the distribution of $G$-concurrence has been calculated in [20]; the average von Neumann entropy had been obtained in 21]; the moments for the purity distribution in random states were calculated analytically in [22, as well as approximate moments of Meyer-Wallach entanglement (a multipartite entanglement measure based on the purity). The aim of this paper is to provide analytic expressions for the probability density distribution of the purity (or the linear entropy) for bipartite random pure states. Explicit analytical formulae are derived for the smallest bipartitions of the Hilbert space into a $p$-dimensional and a $q$-dimensional spaces: $p=q=3$ in Section 3 , 
$p=3$ and any $q$ in Section 4. $p=q=4$ in Section 5 A different method allowing to obtain formulae for $p=4$ and $q \geq 4$ is explained in Section 5 The method is general, and similar calculations would yield expressions for higher values of $p$ and $q$, although it is not sure whether these formulae would take any nice and compact form.

\section{Probability density distribution of the purity.}

Let us consider a state $\Psi$ belonging to the Hilbert space $\mathbb{C}^{p} \otimes \mathbb{C}^{q}$ for some integers $p, q$ with $p \leq q$. Suppose $\Psi$ admits the following Schmidt decomposition 23.

$$
\Psi=\sum_{i=1}^{p} \sqrt{x_{i}}\left|a_{i}\right\rangle \otimes\left|b_{i}\right\rangle
$$

where $\left|a_{i}\right\rangle$ and $\left|b_{i}\right\rangle, 1 \leq i \leq p$, are respectively orthonormal bases for $\mathbb{C}^{p}$ and $\mathbb{C}^{q}$. The purity $R$ of the state $\Psi$ can be written in terms of Schmidt coefficients as

$$
R(\Psi)=\sum_{i=1}^{p} x_{i}^{2}
$$

The linear entropy is expressed in terms of the purity by the simple relation

$$
S_{L}(\Psi)=\frac{p}{p-1}(1-R(\Psi)) .
$$

For random pure states obtained as column vector of random matrices distributed according to the unitarily invariant Haar measure (CUE matrices), the Schmidt coefficients are characterized by the following joint distribution [19]:

$$
P\left(x_{1}, \ldots, x_{p}\right)=\mathcal{A} \prod_{1 \leq i<j \leq p}\left(x_{i}-x_{j}\right)^{2} \prod_{1 \leq i \leq p} x_{i}^{q-p} \delta\left(1-\sum_{i=1}^{p} x_{i}\right)
$$

for $x_{i} \in[0,1] ; \delta$ is the Dirac delta function, and $\mathcal{A}$ is the normalisation factor

$$
\mathcal{A}=\frac{(p q-1) !}{\prod_{0 \leq j \leq p-1}(q-j-1) !(p-j) !} .
$$

The purity distribution function is then given by

$$
P(R)=\mathcal{A} \int_{0}^{1} d^{p} \mathbf{x} V(\mathbf{x})^{2} \prod_{1 \leq i \leq p} x_{i}^{q-p} \delta\left(1-\sum_{i=1}^{p} x_{i}\right) \delta\left(R-\sum_{i=1}^{p} x_{i}^{2}\right),
$$

where we have introduced the Vandermonde determinant $V(\mathbf{x})=\prod_{i<j}\left(x_{i}-x_{j}\right)$ with $\mathbf{x}=\left(x_{1}, \ldots, x_{p}\right)$. The distribution $\tilde{P}\left(S_{L}\right)$ of the linear entropy can be straightforwardly deduced from $P(R)$ using Eq. (3):

$$
\tilde{P}\left(S_{L}\right)=\frac{p-1}{p} P\left(1-\frac{p-1}{p} S_{L}\right) .
$$

As the purity vanishes outside the interval $[1 / p, 1]$, the distribution of linear entropy is supported by $[0,1]$.

In the next sections we evaluate (6) in the cases $p=2,3,4$. We first note that the expression (6) can be simplified to

$$
P(R)=\mathcal{A} p ! \int_{0}^{1} d^{p} \mathbf{x} V(\mathbf{x}) \prod_{1 \leq i \leq p} x_{i}^{d+k-1} \delta\left(1-\sum_{i=1}^{p} x_{i}\right) \delta\left(R-\sum_{i=1}^{p} x_{i}^{2}\right),
$$

where $d=q-p$, using a transformation detailed in [22]. 


\section{Distribution of the purity for a $2 \times q$ bipartite random state.}

In the case $p=2, q \geq 2$, the analytic expression for the probability distribution $P(R)$ can easily be obtained analytically directly by integration of (6). It reads

$$
P(R)=\frac{(2 q-1) !}{2^{q-1}(q-1) !(q-2) !}(1-R)^{q-2} \sqrt{2 R-1}
$$

for $1 / 2 \leq R \leq 1$, and 0 otherwise.

\section{Distribution of the purity for a $3 \times 3$ bipartite random state.}

In the case $p=3$ and $q=3$, Eq. (6) reads

$$
P(R)=\frac{8 !}{4} \int_{0}^{1} d^{3} \mathbf{x} V(\mathbf{x}) x_{2} x_{3}^{2} \delta\left(1-\sum_{i=1}^{3} x_{i}\right) \delta\left(R-\sum_{i=1}^{3} x_{i}^{2}\right) .
$$

The domain of integration is the intersection of the cube $[0,1]^{3}$, the plane $x_{1}+x_{2}+x_{3}=$ 1 and the sphere of radius $\sqrt{R}$ centered on the origin. This intersection is a circle if $\frac{1}{3} \leq R \leq \frac{1}{2}$ (see Fig. [1 left), or sections of a circle if $\frac{1}{2} \leq R \leq 1$. We first make the change of variables $\mathbf{x}=O \mathbf{X}$, where $O$ is the orthogonal matrix

$$
\left(\begin{array}{ccc}
-\frac{2}{\sqrt{6}} & 0 & \frac{1}{\sqrt{3}} \\
\frac{1}{\sqrt{6}} & -\frac{1}{\sqrt{2}} & \frac{1}{\sqrt{3}} \\
\frac{1}{\sqrt{6}} & \frac{1}{\sqrt{2}} & \frac{1}{\sqrt{3}}
\end{array}\right)
$$

In the new coordinates $\left(X_{1}, X_{2}, X_{3}\right)$, the plane has equation $X_{3}=1 / \sqrt{3}$ and the sphere $X_{1}^{2}+X_{2}^{2}+X_{3}^{2}=R$. We first integrate over $X_{3}$ and make a change of variables to polar coordinates $\left(X_{1}=\rho \cos \theta, X_{2}=\rho \sin \theta\right)$. Let us set $\phi=0$ if $\frac{1}{3} \leq R \leq \frac{1}{2}$, $\phi=\arccos (1 / \sqrt{6 R-2})$ if $\frac{1}{2} \leq R \leq 1$, and $r=\sqrt{R-1 / 3}$. The domain of integration in the plane $X_{3}=1 / \sqrt{3}$ is represented in Fig. 1 right for two different values of $R$. After integrating over $\rho$ we obtain

$$
\begin{aligned}
P(R) & =-\frac{7 ! r^{3}}{\sqrt{6}} \int_{\mathcal{D}_{\phi}} d \theta\left(\frac{1}{3}-r \sqrt{\frac{2}{3}} \cos \left(\theta+\frac{4 \pi}{3}\right)\right) \\
& \times\left(\frac{1}{3}-r \sqrt{\frac{2}{3}} \cos \left(\theta+\frac{2 \pi}{3}\right)\right)^{2} \sin (3 \theta) .
\end{aligned}
$$

The domain of integration for $\theta$ is $\mathcal{D}_{\phi}=[0,2 \pi] \backslash \mathcal{C}_{\phi}$, where

$$
\mathcal{C}_{\phi}=[-\phi, \phi] \bigcup\left[-\phi+\frac{2 \pi}{3}, \phi+\frac{2 \pi}{3}\right] \bigcup\left[-\phi+\frac{4 \pi}{3}, \phi+\frac{4 \pi}{3}\right] .
$$

The integrand in Eq. (12) can be expanded as a sum of $\cos k \theta$ and $\sin k \theta$. Terms of the form $\sin k \theta$ yield zero upon integration; terms of the form $\cos k \theta$ yield

$$
\int_{\mathcal{C}_{\phi}} d \theta \cos k \theta=\int_{-\phi}^{\phi} d \theta\left(1+\cos \frac{2 k \pi}{3}+\cos \frac{4 k \pi}{3}\right) \cos k \theta
$$

which is zero unless $3 \mid k$. Nonzero contributions therefore necessarily come from the $\cos \left(\theta+\frac{4 \pi}{3}\right) \cos ^{2}\left(\theta+\frac{2 \pi}{3}\right) \sin (3 \theta)$ term in (12). Keeping only terms of the form $\cos 3 k t$ in the expansion of this term we get

$$
P(R)=70 \sqrt{3} r^{6}(2 \pi-6 \phi+\sin (6 \phi)) .
$$


Replacing $\phi$ and $R$ by their value we finally obtain

$$
P(R)=70 \sqrt{3} \mid \begin{array}{ll}
2 \pi\left(R-\frac{1}{3}\right)^{3}, & \frac{1}{3} \leq R \leq \frac{1}{2} \\
6\left(R-\frac{1}{3}\right)^{3}\left(\frac{\pi}{3}-\arccos \frac{1}{\sqrt{6 R-2}}\right) & \\
\quad+(R-1)\left(R-\frac{5}{9}\right) \sqrt{6 R-3}, & \frac{1}{2} \leq R \leq 1 .
\end{array}
$$

It is interesting to check whether this formula allows to recover the moments derived in [22]. The value of $\left\langle R^{n}\right\rangle$ is given (see [22]) by

$$
\begin{aligned}
& \left\langle R^{n}\right\rangle=\frac{p !(p q-1) !}{(p q+2 n-1) !} \sum_{n_{1}+n_{2}+\cdots+n_{p}=n} \frac{n !}{n_{1} ! n_{2} ! \ldots n_{p} !} \\
& \times \prod_{i=1}^{p} \frac{\left(q+2 n_{i}-i\right) !}{(q-i) ! i !} \prod_{1 \leq i<j \leq p}\left(2 n_{i}-i-2 n_{j}+j\right)
\end{aligned}
$$

(correcting by a factor $p$ ! the Eq. (10) of [22, where this term had been erroneously forgotten). The first moments of $P(R)$ as calculated from Eq. (16) yield values that are precisely equal to those obtained from Eq. (17).
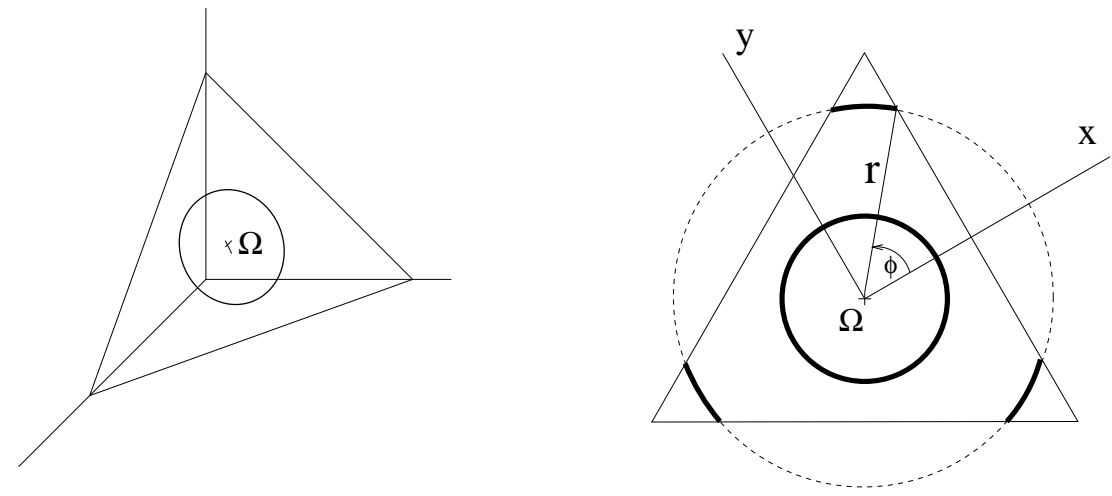

Figure 1. Domain of integration for $p=3$. Representation in $\mathbb{R}^{3}$ for $\frac{1}{3} \leq R \leq \frac{1}{2}$ (left), and in the plane $x_{1}+x_{2}+x_{3}=1$ (right, thick lines).

\section{Distribution of the purity for a $3 \times q$ bipartite random state, $q \geq 3$.}

In the case of a random vector belonging to a Hilbert space of dimensions $p \times q$ with $p=3$ and $q \geq 3$, the term $V\left(x_{1}, x_{2}, x_{3}\right) x_{2} x_{3}^{2}$ in (10) is multiplied by a factor $\left(x_{1} x_{2} x_{3}\right)^{d}$, where $d=q-p$. The changes of variables of Section 3 yield (still setting $r=\sqrt{R-1 / 3})$ an integrand that can be expanded as

$$
\begin{gathered}
-\frac{r^{3}}{\sqrt{2}} \sum_{k=0}^{d}\left(\begin{array}{l}
d \\
k
\end{array}\right) \\
\left(\frac{1}{27}-\frac{r^{2}}{6}\right)^{d-k}\left(-\frac{r^{3}}{3 \sqrt{6}}\right)^{k}\left(\frac{1}{3}-r \sqrt{\frac{2}{3}} \cos \left(\theta+\frac{4 \pi}{3}\right)\right) \\
\times\left(\frac{1}{3}-r \sqrt{\frac{2}{3}} \cos \left(\theta+\frac{2 \pi}{3}\right)\right)^{2} \sin 3 \theta \cos ^{k} 3 \theta
\end{gathered}
$$

Again in (18) only terms of the form $\cos (3 j \theta)$ contribute to the final result, and nonzero contributions therefore necessarily come from the

$$
f_{k}(\theta)=\cos \left(\theta+\frac{4 \pi}{3}\right) \cos ^{2}\left(\theta+\frac{2 \pi}{3}\right) \sin (3 \theta) \cos ^{k} 3 \theta
$$


term. For a given $k$, the expansion of $\cos ^{k} 3 \theta$ yields

$$
\cos ^{k} 3 \theta= \begin{cases}\frac{1}{2^{k-1}} \sum_{j=0}^{k / 2}\left(1-\frac{1}{2} \delta_{j}\right)\left(\begin{array}{c}
k \\
k / 2-j
\end{array}\right) \cos 6 j \theta & k \text { even } \\
\frac{1}{2^{k-1}} \sum_{j=0}^{(k-1) / 2}\left(\begin{array}{c}
k \\
(k-1) / 2-j
\end{array}\right) \cos (6 j+3) \theta & k \text { odd },\end{cases}
$$

where $\delta_{j}$ is the Kronecker delta. For a fixed integer $t$ the only terms contributing to $P(R)$ in the expansion of $\cos \left(\theta+\frac{4 \pi}{3}\right) \cos ^{2}\left(\theta+\frac{2 \pi}{3}\right) \sin (3 \theta) \cos 3 t \theta$ sum up to $\sqrt{3}(\cos (3 t-6) \theta-2 \cos 3 t \theta+\cos (3 t+6) \theta) / 32$. The integral of $f_{k}(\theta)$ over $\mathcal{C}_{\phi}$ thus yields

$$
\begin{aligned}
& \frac{3 \sqrt{3}}{2^{k+3}} \sum_{j=0}^{k / 2}\left(1-\frac{1}{2} \delta_{j}\right)\left(\begin{array}{c}
k \\
k / 2-j
\end{array}\right) \chi_{j}(\phi), \quad k \text { even } \\
& \frac{3 \sqrt{3}}{2^{k+3}} \sum_{j=0}^{(k-1) / 2}\left(\begin{array}{c}
k \\
(k-1) / 2-j
\end{array}\right) \chi_{j+1 / 2}(\phi), \quad k \text { odd },
\end{aligned}
$$

where we have defined, for $j \geq 0$, the function

$$
\chi_{j}(\phi)=\frac{\sin (6 j-6) \phi}{6 j-6}-2 \frac{\sin 6 j \phi}{6 j}+\frac{\sin (6 j+6) \phi}{6 j+6}
$$

(for $j=0$ and $j=1$ it is understated that the limit $j \rightarrow 0$ or $j \rightarrow 1$ is taken). Summing all contributions together we finally obtain the exact expression

$$
\begin{aligned}
& P(R)=\frac{(3 q-1) !}{16 \sqrt{3} \prod_{j=1}^{3}(q-j) !} \sum_{k=0}^{d}\left(\begin{array}{l}
d \\
k
\end{array}\right)\left(\frac{-1}{6 \sqrt{6}}\right)^{k}\left(\frac{5-9 R}{54}\right)^{d-k}\left(R-\frac{1}{3}\right)^{\frac{3}{2}(k+2)} \\
& \times \sum_{j=0}^{\lfloor k / 2\rfloor}\left(1-\frac{1}{2} \delta_{j} \delta_{\bar{k}}\right)\left(\begin{array}{c}
k \\
\left\lfloor\frac{k}{2}\right\rfloor-j
\end{array}\right)\left(\chi_{j+\bar{k} / 2}(\phi)-\chi_{j+\bar{k} / 2}\left(\frac{\pi}{3}\right)\right),
\end{aligned}
$$

with again $\phi=0$ for $1 / 3 \leq R \leq 1 / 2$ and $\arccos (1 / \sqrt{6 R-2})$ for $1 / 2 \leq R \leq 1$. Here $\lfloor x\rfloor$ is the integer part of $x, \bar{k}=k \bmod 2$ and $\delta$ is the Kronecker delta symbol. Note that the term $\chi_{j+\bar{k}}(\phi)$ can be expressed as a function of $R$ in terms of Chebychev polynomials of the second kind $U_{n}$, using the relation $\sin n \theta=U_{n-1}(\cos \theta) \sin \theta$. Again, on can check that the expression (24) allows to recover the moments (17).

\section{Distribution of the purity for a $4 \times 4$ bipartite random state.}

The treatment of the $p=4$ case is quite similar to the previous one but calculations (and results) quickly get very heavy. Here we derive an explicit expression for the case $p=q=4$. The first steps of Section 3 are easily generalized (they can in fact be generalized in an obvious way to arbitrary $p, q$ ). The domain of integration in (6) is the intersection of a plane and a hypersphere in $\mathbb{R}^{4}$, restricted to $[0,1]^{4}$. First we make a change of variables $\mathbf{x}=O \mathbf{X}$, where $O$ is the orthogonal matrix

$$
\left(\begin{array}{cccc}
-\frac{3}{\sqrt{12}} & 0 & 0 & \frac{1}{2} \\
\frac{1}{\sqrt{12}} & -\frac{2}{\sqrt{6}} & 0 & \frac{1}{2} \\
\frac{1}{\sqrt{6}} & \frac{1}{\sqrt{6}} & -\frac{1}{\sqrt{2}} & \frac{1}{2} \\
\frac{1}{\sqrt{6}} & \frac{1}{\sqrt{6}} & \frac{1}{\sqrt{2}} & \frac{1}{2}
\end{array}\right)
$$


so that the plane has equation $X_{4}=1 / 2$ and the sphere $X_{1}^{2}+X_{2}^{2}+X_{3}^{2}+X_{4}^{2}=R$. After integration over $X_{4}$, we have to integrate

$$
f\left(X_{1}, X_{2}, X_{3}\right)=x_{2} x_{3}^{2} x_{4}^{3} V\left(x_{1}, x_{2}, x_{3}, x_{4}\right)
$$

over the portion of the sphere of radius $r=\sqrt{R-1 / 4}$ that is comprised inside the thetrahedron of vertices $V_{1}=(-\sqrt{3} / 2,0,0), V_{2}=(1 / \sqrt{12},-2 / \sqrt{6}, 0), \quad V_{3}=$ $(1 / \sqrt{12}, 1 / \sqrt{6},-1 / \sqrt{2})$ and $V_{4}=(1 / \sqrt{12}, 1 / \sqrt{6}, 1 / \sqrt{2})$, centered on $(0,0,0)$. In the case $1 / 4<R<1 / 3$, the sphere is entirely inside the tetrahedron: after making a change of variables to spherical coordinates $\left(X_{1}=\rho \cos \theta_{1}, X_{2}=\rho \sin \theta_{1} \cos \theta_{2}, X_{3}=\right.$ $\left.\rho \sin \theta_{1} \sin \theta_{2}\right)$, the integration is trivial. Taking into account the factors $1 / 2$ and $1 / 2 r$ coming from the integration of $\delta\left(2 X_{4}-1\right)$ and $\delta\left(R-1 / 4-\rho^{2}\right)$ the integration of (26) yields

$$
\int d \mathbf{X} f(\mathbf{X})=4 \pi r^{13} / 45045
$$

with $\mathbf{X}=\left(X_{1}, X_{2}, X_{3}\right)$. When $1 / 3<R<1 / 2$, the integrand can first be simplified by use of the symmetries of the domain of integration, which consists of the sphere minus the four spherical caps emerging from the tetrahedron. We note $C_{i}, 1 \leq i \leq 4$ the cap opposite to vertex $V_{i}$. Let $R_{i}(\varphi)$ be the rotation of axis $O X_{i}, i=1,2,3$, and angle $\varphi$. The caps $C_{2}, C_{3}, C_{4}$ can be obtained from $C_{1}$ by rotations: $C_{2}$ is the image of $C_{1}$ by $S=R_{1}(-\pi / 3) R_{3}(\arccos (-1 / 3))$, and $C_{3}$ and $C_{4}$ are respectively the images of $C_{2}$ by $T=R_{1}(2 \pi / 3)$ and $T^{2}=R_{1}(4 \pi / 3)$. Therefore

$$
\int_{\bigcup_{i=1}^{4} C_{i}} d \mathbf{X} f(\mathbf{X})=\int_{C_{1}} d \mathbf{X}\left(f(\mathbf{X})+f(S \mathbf{X})+f(T S \mathbf{X})+f\left(T^{2} S \mathbf{X}\right)\right) .
$$

In spherical coordinates, the top cap (cap $C_{1}$ ) has equation $\rho=r, 0 \leq \theta_{1} \leq$ $\arccos \frac{1}{2 r \sqrt{3}}, 0 \leq \theta_{2} \leq 2 \pi$ (we recall that $r=\sqrt{R-1 / 4}$ ). Performing the integrals over $\theta_{2}$, and then $\theta_{1}$, in (28) yields

$$
\begin{array}{r}
\int d \mathbf{X} f(\mathbf{X})=\frac{967 \pi}{804925734912 \sqrt{3}}-\frac{571 \pi}{9459597312 \sqrt{3}} r^{2}+\frac{505 \pi}{429981696 \sqrt{3}} r^{4} \\
-\frac{229 \pi}{20901888 \sqrt{3}} r^{6}+\frac{\pi}{20480 \sqrt{3}} r^{8}-\frac{11 \pi}{124416 \sqrt{3}} r^{10}-\frac{\pi}{20736 \sqrt{3}} r^{12}+\frac{8 \pi}{45045} r^{13} .
\end{array}
$$

Finally, when $1 / 2<R<1$, the domain of integration can be further reduced to $\theta_{2} \in[0, \pi / 3]$ by applying $T$ and $T^{2}$ and using the symmetry $\theta_{2} \rightarrow-\theta_{2}$. The domain of integration is $\theta_{2} \in[0, \pi / 3]$ and $\theta_{1} \in[\varphi, \pi[$ where $\varphi$ verifies

$$
\cos \theta_{2}=\frac{r \cos \varphi+\sqrt{3} / 2}{2 \sqrt{2} r \sin \varphi}
$$

and the integrand is a sum of terms of the form $\cos ^{2 k} \theta_{1} \sin \theta_{1} \cos ^{2 k^{\prime}} \theta_{2}$ and $\cos ^{2 k+1} \theta_{1} \cos ^{2 k^{\prime}+1} \theta_{2}$. The calculation gets tedious and the steps leading to $P(R)$ in this case are given in the Appendix.

Taking into account the normalization factor $\mathcal{A} p !=15 ! /(3 ! 2 !)^{2}$ we finally get $P(R)=0$ for $R \notin[1 / 4,1]$ and

$$
\begin{array}{ll}
P(R)=\frac{1575 \pi}{16}(4 R-1)^{\frac{13}{2}}, & R \in\left[\frac{1}{4}, \frac{1}{3}\right] \\
P(R)=\frac{\pi}{3} Q_{2}(R)-\frac{1575 \pi}{16}(4 R-1)^{\frac{13}{2}}, & R \in\left[\frac{1}{3}, \frac{1}{2}\right]
\end{array}
$$




$$
\begin{aligned}
P(R) & =\sqrt{6 R-3} Q_{1}(R)+\left(\frac{\pi}{3}-\arccos \left(\frac{1}{\sqrt{6 R-2}}\right)\right) Q_{2}(R) \\
& -\frac{4725}{16}(4 R-1)^{\frac{13}{2}}\left(\frac{\pi}{3}-\arccos \left(\frac{R}{3 R-1}\right)\right), R \in\left[\frac{1}{2}, 1\right]
\end{aligned}
$$

where we have introduced the following two polynomials $Q_{1}(x)=\frac{175}{1944 \sqrt{3}}(1-$ $x)\left(1657+277731 x-2190321 x^{2}+6208416 x^{3}-7386066 x^{4}+2913408 x^{5}\right)$ and $Q_{2}(x)=$ $\frac{175}{1944 \sqrt{3}}\left(-159241+2178306 x-11709126 x^{2}+30254796 x^{3}-34540506 x^{4}+4864860 x^{5}+\right.$ $\left.14594580 x^{6}\right)$. Again it can be checked analytically that the moments obtained from the above formula for $P(R)$ agree with those obtained from Eq. (17).

\section{Probability density distribution of the purity for a $4 \times q$ bipartite random pure state, $q \geq 4$.}

Similar calculations can be done in the same way for $q>4$. However as the number of terms in the integrand gets large, it is more efficient to proceed in the following way. The only difference between the $q=4$ and the $q>4$ cases is that the integrand is multiplied by $\left(x_{1} x_{2} x_{3} x_{4}\right)^{d}$, where $d=q-p$. It is then easy to see from Section 5 that the integrals appearing in the calculation for $1 / 4<R<1 / 3$ and $1 / 3<R<1 / 2$ still yield polynomials in $r$. For $1 / 2<R<1$, one can show that after reducing the domain of integration by symmetries as in the previous section, the integrand is again a sum of terms of the form $\cos ^{2 k} \theta_{1} \sin \theta_{1} \cos ^{2 k^{\prime}} \theta_{2}$ and $\cos ^{2 k+1} \theta_{1} \cos ^{2 k^{\prime}+1} \theta_{2}$. The calculation therefore yields an expression similar to Eq. (35), but with polynomials in $r$ of higher degree. Therefore one can look for a $P(R)$ (expressed in terms of $r=\sqrt{R-1 / 4}$ ) of the form $A_{5}(r)$ for $1 / 4 \leq R \leq 1 / 3, A_{6}(r)$ for $1 / 3 \leq R \leq 1 / 2$, and of the form of Eq. (35) for $1 / 2 \leq R \leq 1$, where $A_{i}$ all are polynomials in $r$. The maximal order of the $A_{i}$ corresponds to the order of the integrand $\left(x_{1} x_{2} x_{3} x_{4}\right)^{d} x_{2} x_{3}^{2} x_{4}^{3} V\left(x_{1}, x_{2}, x_{3}, x_{4}\right)$, that is (taking into account the $r^{2}$ from the Jacobian and the $1 / r$ of the integration of one of the delta functions) $13+4 d$. The coefficients of these polynomials are unknown variables. The knowledge of all moments (17) allows us to write down as many linear equations as there are unknown variables, that is $6(14+4 d)$. The problem now reduces to solving a linear system $M x=b$. The vector $b=\left\{\left\langle R^{0}\right\rangle,\left\langle R^{1}\right\rangle,\left\langle R^{2}\right\rangle, \ldots\right\}$ is obtained from (17), and $M$ is a matrix of size $6(14+4 d)$ whose coefficients are given by terms of the form

$$
\int_{\sqrt{1 / k-1 / 4}}^{\sqrt{1 /(k-1)-1 / 4}} r^{\nu} g(r) d r, \quad k=2,3,4,
$$

where $g(r)$ is one of the functions $1, \arctan \sqrt{\frac{8 r^{2}}{4 r^{2}-1}}, \arctan \sqrt{\frac{2}{12 r^{2}-3}}$ or $\sqrt{4 r^{2}-1}$. These integrals can be calculated analytically. The solution $x=M^{-1} b$ of the system yields the coefficients of the polynomials $A_{i}$ and thus an analytic formula for $P(R)$.

A similar method would yield expressions for higher Hilbert space dimensions. However, given what Eq. (31) looks like, it is not clear whether formulae for higher dimensions could be cast into a tractable form.

The author thanks CalMiP in Toulouse and Idris in Orsay for access to their supercomputers, and Phuong Mai Dinh for helpful comments. This work was supported by the Agence Nationale de la Recherche (ANR project INFOSYSQQ) and the European program EuroSQIP. 


\section{Appendix}

The derivation of $P(R)$ for $p=4, q=4$ and $\frac{1}{2} \leq R \leq 1$ starts from the integrand obtained after having reduced the domain of integration by symmetries, and integrated over $\rho$ and $\theta_{1} \in\left[\varphi, \pi\left[\right.\right.$. From Eq. (30) and using the fact that $\theta_{2} \in[0, \pi / 3[$ we obtain an expression for $\sin \varphi$ and $\cos \varphi$ which allows to express the integrand as a function of $\theta_{2}$ only. Expanding all trigonometric terms and changing variables from $\cos \theta_{2}$ to $x$, we are left with a sum of terms of the form

$$
\frac{x^{2 a} \sqrt{1-x^{2}}}{\left(1+8 x^{2}\right)^{13}}
$$

and

$$
\frac{x^{2 a+1} \sqrt{1-x^{2}} \sqrt{4 r^{2}\left(1+8 x^{2}\right)-3}}{\left(1+8 x^{2}\right)^{13}},
$$

where $a$ is some integer and $x$ has to be integrated between $1 / 2$ and 1 . The integrals corresponding to (33) give constants independent of $r$. The integrals corresponding to (34) can be evaluated by the change of variables $t=\sqrt{\left(x^{2}-c\right) /\left(1-x^{2}\right)}$ with $c=\left(3-4 r^{2}\right) /\left(32 r^{2}\right)$. Upon integration, we obtain terms of the form

$$
A_{1}(r)+A_{2}(r) \arctan \sqrt{\frac{8 r^{2}}{4 r^{2}-1}}+A_{3}(r) \arctan \sqrt{\frac{2}{12 r^{2}-3}}+A_{4}(r) \sqrt{4 r^{2}-1},
$$

where $A_{i}$ are polynomials in $r$. Replacing $r$ by its value $\sqrt{R-\frac{1}{4}}$ and noting that

$$
\begin{aligned}
& \arccos \sqrt{\frac{2}{12 r^{2}-1}}+\arctan \sqrt{\frac{2}{12 r^{2}-3}}=\frac{\pi}{2} \\
& \frac{1}{2} \arccos \frac{4 r^{2}+1}{12 r^{2}-1}+\arctan \sqrt{\frac{8 r^{2}}{4 r^{2}-1}}=\frac{\pi}{2},
\end{aligned}
$$

we get the final result (31).

[1] D. P. DiVincenzo, D. W. Leung, and B. M. Terhal, IEEE Trans. Inf. Theory 48 (3), 580-598 (2002).

[2] A. Harrow, P. Hayden, and D. Leung, Phys. Rev. Lett. 92, 187901 (2004).

[3] P. Zanardi, C. Zalka, and L. Faoro, Phys. Rev. A 62, 030301 (2000).

[4] J. N. Bandyopadhyay and A. Lakshminarayan, Phys. Rev. Lett. 89, 060402 (2002).

[5] A.J. Scott, Physical Review A 69, 052330 (2004).

[6] O. Giraud and B. Georgeot, Phys. Rev. A 72, 042312 (2005).

[7] Y. S. Weinstein and C. S. Hellberg, Phys. Rev. Lett. 95, 030501 (2005).

[8] M. Znidaric, arxiv/quant-ph/0702240.

[9] M. B. Plenio and S. Virmani, Quant. Inf. Comput. 7, 1 (2007).

[10] C. H. Bennett, H. Bernstein, S. Popescu, and B. Schumacher, Phys. Rev. A 53, 2046 (1996).

[11] R. Demkowicz-Dobrzanski and M. Kus, Phys. Rev. E 70, 066216 (2004).

[12] R. F. Abreu and R. O. Vallejos, Phys. Rev. A 75, 062335 (2007).

[13] P. Facchi, G. Florio and S. Pascazio, Phys. Rev. A 74, 042331 (2006).

[14] M. Znidaric and T. Prosen, Phys. Rev. A 71, 032103 (2005).

[15] O. Giraud, J. Martin and B. Georgeot, arXiv:0704:2765 (2007).

[16] L. Viola and W. G. Brown, J. Phys. A 40, 8109 (2007).

[17] A. Hurwitz, Nachr. Ges. Wiss. Goettinger Math. Phys. Kl. 71, (1897).

[18] M. L. Mehta, Random Matrices (Academic Press, New York, 1991).

[19] K. Zyczkowski and H. -J. Sommers, J. Phys. A: Math. Gen. 34, 7111-7125 (2001).

[20] V. Cappellini, H. -J. Sommers and K. Zyczkowski, Phys. Rev. A 74, 062322 (2006).

[21] D. N. Page, Phys. Rev. Lett. 71, 1291 (1993).

[22] O. Giraud, J. Phys. A 40, 2793 (2007).

[23] M. A. Nielsen and I. L. Chuang, Quantum computation and quantum information, Cambridge university press (2000). 